\title{
Treatment of spinal muscular atrophy cells with drugs that upregulate SMN expression reveals inter- and intra-patient variability
}

\author{
Eva Also-Rallo ${ }^{1}$, Laura Alías ${ }^{1}$, Rebeca Martínez-Hernández ${ }^{1}$, Lidia Caselles ${ }^{1}$, María J Barceló ${ }^{1}$, \\ Montserrat Baiget ${ }^{1}$, Sara Bernal ${ }^{1}$ and Eduardo F Tizzano ${ }^{\star, 1}$
}

Spinal muscular atrophy (SMA) is a genetic neuromuscular disorder caused by mutations in the SMN1 gene. The homologous copy (SMN2) is always present in SMA patients. SMN1 gene transcripts are usually full-length (FL), but exon 7 is spliced out in a high proportion of SMN2 transcripts (delta7) ( $\Delta 7$ ). Advances in drug therapy for SMA have shown that an increase in SMN mRNA and protein levels can be achieved in vitro. We performed a systematic analysis of $S M N$ expression in primary fibroblasts and EBV-transformed lymphoblasts from seven SMA patients with varying clinical severity and different SMN1 genotypes to determine expression differences in two accessible tissues (skin and blood). The basal expression of SMN mRNA FL and $\triangle 7$ in fibroblasts and lymphoblasts was analyzed by quantitative real-time PCR. The FL-SMN and FL/A7 SMN ratios were higher in control cells than in patients. Furthermore, we investigated the response of these cell lines to hydroxyurea, valproate and phenylbutyrate, drugs previously reported to upregulate SMN2. The response to treatments with these compounds was heterogeneous. We found both intra-patient and inter-patient variability even within haploidentical siblings, suggesting that tissue and individual factors may affect the response to these compounds. To optimize the stratification of patients in clinical trials, in vitro studies should be performed before enrolment so as to define each patient as a responder or non-responder to the compound under investigation.

European Journal of Human Genetics (2011) 19, 1059-1065; doi:10.1038/ejhg.2011.89; published online 25 May 2011

Keywords: SMA; SMN; hydroxyurea; valproic acid; phenylbutyrate

\section{INTRODUCTION}

Spinal muscular atrophy (SMA) is caused by degeneration of the spinal cord $\alpha$-motor neurons, leading to muscle denervation and atrophy. SMA is the second most common inherited lethal disorder of childhood. It is classified into four types (I-IV), depending on age at onset and disease severity. Type I SMA (Werdnig-Hoffmann disease) is the most severe form of the disease, with clinical onset usually occurring before 6 months of age, whereas type II SMA is the intermediate form with onset between 6 and 18 months. Type III SMA (Kugelberg-Welander disease) is a relatively mild form of the disease, with onset usually occurring in childhood or adolescence. Type IV SMA appears in adult life and clinical manifestations may be minimal. Mutations or deletions in the survival motor neuron gene, SMN1, have been found to be the cause of SMA. ${ }^{1,2}$ In humans, two highly homologous copies of the survival motor neuron genes (the telomeric SMN1 and the centromeric SMN2) are located in a duplicated region of chromosome $5 \mathrm{q}_{13} .^{2}$ Over $95 \%$ of $5 \mathrm{q}$-linked SMA patients have homozygous deletions for SMN1 or gene conversion at exons 7 or 8 , whereas the remaining subjects have missense, nonsense or splice site mutations. ${ }^{1}$ A 4 -bp deletion in exon 3 (c.399_402del AGAG) - exclusive to the Spanish population as far as we know - constitutes one of the most frequently found subtle mutations in SMA. It has been associated with a large spectrum of phenotypes, from severe type I to non-symptomatic patients. ${ }^{3-5}$
The SMN1 gene contains a C nucleotide at position six of exon 7 $(E x 7+6)$ and produces predominantly full-length transcripts (FL-SMN). The SMN2 gene, however, contains a $\mathrm{T}$ nucleotide at this position, leading to a differentially spliced form that lacks exon 7 $(\Delta 7-S M N) .^{2,6-8}$ The resulting putative shorter protein has a different C-terminus. It is less stable and cannot oligomerize or self-associate, as occurs with the complete protein., ${ }^{9,10}$

Patients with the type I form usually have a lower number of SMN2 copies than the chronic types II-IV. However, the correlation is not absolute and patients with three SMN2 copies may suffer type I, II or III SMA. ${ }^{11,12}$

As the differences between SMN1 and SMN2 are related to the complete transcript and the amount of protein, drugs capable of increasing FL-SMN expression and SMN protein may have therapeutic effects for SMA patients. ${ }^{13}$ Histone deacetylases inhibitors (HDACi), for example, increase acetylation of histones and other proteins ${ }^{14}$ and this hyperacetylation relaxes the tertiary structure of chromatin, facilitating access of the transcriptional machinery to target genes. In vitro experiments with phenylbutyrate (PBA) and valproic acid (VPA) - two well-known HDACi - have shown an increase in SMN mRNA and protein levels in SMA fibroblasts. ${ }^{15-17}$ Similar studies with hydroxyurea (HU) in EBV-immortalized SMA lymphoblasts ${ }^{18}$ have shown an increase in the FL-SMN/D7-SMN (FL/ $\Delta 7$ ratio). Pilot trials with these drugs have been performed in SMA patients and results 
were promising, ${ }^{19-21}$ leading to the development of placebo-controlled clinical trials. PBA has been investigated in a double-blind placebocontrolled trial in 107 children with type II SMA. VPA-carnitine has been administered to 42 type II SMA children in a multi-centre phase II trial. ${ }^{22}$ A double-blind placebo-controlled trial with HU in 28 type II SMA and 29 type III SMA patients has recently been completed. ${ }^{23}$ Results from these three clinical trials have not revealed a clear benefit of these drugs for the patients.

To detect possible differences in individual responses in a group of SMA patients with diverse genotypes and phenotypes, we analyzed SMN mRNA and protein levels in response to HU, VPA and PBA in two cell types (fibroblasts and lymphoblasts). In particular, we aimed to compare the responses of four sisters, born to consanguineous parents, who are homozygous for a frameshift mutation in the SMN1 gene and have the same SMN2 copy number but discordant phenotypes.

\section{MATERIALS AND METHODS}

\section{Patients and cell cultures}

A total of 10 individuals (3 controls and 7 SMA) were included in this study. SMA was diagnosed using the criteria outlined by the International SMA Consortium, ${ }^{24}$ and confirmed by detection of molecular alterations in the SMN1 gene. SMN1 genotype and SMN2 copy number were determined as previously described. ${ }^{1,11}$ Informed consent was obtained from all subjects or their parents. Human fibroblasts and EBV-immortalized lymphoblasts were cultured according to standard protocols. RNA analysis was performed at 8 and $24 \mathrm{~h}$ after feeding for PBA and after 24 and $48 \mathrm{~h}$ for VPA and HU. Table 1 shows patients' characteristics. Four sisters (patients 4-7) were homozygous for a frameshift mutation in exon 3 with four copies of SMN2. They were haploidentical for the SMA locus sharing the region comprised between markers rs4613646 and rs13162495 (12 Mb) (Also-Rallo et al, unpublished data). One of the sisters, patient 7, is able to walk, whereas her three sisters have been wheelchair-confined since early infancy.

\section{Drugs}

In accordance with previously described optimal concentrations, the final concentration of the drugs used in fibroblasts and lymphoblasts was $1 \mathrm{~mm}$ for VPA and HU and $2 \mathrm{~mm}$ for PBA. ${ }^{15,16,18}$ All compounds were freshly prepared immediately before each use. Cells were treated for 24 and $48 \mathrm{~h}$ (with daily addition) for HU and VPA and for 8 and $24 \mathrm{~h}$ for PBA. All treatments were performed in triplicate.

\section{Real-time RT-PCR}

RNA concentration was determined using a spectrophotometer, and $1 \mu \mathrm{g}$ RNA was used for first-strand cDNA synthesis. FL-SMN and $\triangle 7-S M N$ mRNA were

Table 1 Subjects analyzed in this study

\begin{tabular}{lcccc}
\hline Sample & Gender & SMN1 genotype & SMN2 copies & Phenotype \\
\hline C1 & Male & $+/+$ & 2 & Control \\
C2 & Female & $+/+$ & 2 & Control \\
C3 & Female & $+/+$ & 2 & Control \\
1 & Male & Del/Del & 2 & I (Died <2 years) \\
2 & Female & SM/Del & 3 & II (Sitter) \\
3 & Male & Hyb/Del & 3 & III (Sitter) \\
4 & Female & SM/SM & 4 & III (Sitter) \\
5 & Female & SM/SM & 4 & III (Sitter) \\
6 & Female & SM/SM & 4 & III (Sitter) \\
7 & Female & SM/SM & 4 & IV (Walker)
\end{tabular}

Abbreviations: Del, deletion; Hyb, hybrid; SM, subtle mutation c.399 402del AGAG. Patients 4-7 are sisters of consanguineous parents. I-IV refer to the SMA type. determined using GAPDH as an endogenous control in the ABI PRISM 7000 Sequence Detector System (Applied Biosystems, Foster City, CA, USA). All primers, probes and PCR conditions were performed as previously described. ${ }^{15}$ Using the $\Delta \Delta C \mathrm{t}$ method, we analyzed the relative amount of FL-SMN, taking the FL-SMN of each untreated sample as a calibrator (further explained in Figure 3 legend). We also studied the FL/ $\Delta 7$ ratio using the FL-SMN value for each sample as a calibrator.

\section{PCR to determine the origin of SMN transcripts}

Given that the c.399_402del AGAG is a 4-bp deletion that creates a stop codon after $34 \mathrm{bp}$ in the SMN1 gene; FL-SMN levels observed in patients with this mutation should originate only from the SMN2 gene. To confirm this hypothesis, we studied the origin of SMN transcripts amplifying cDNA by using SMN exon 6 (541C618) forward ${ }^{25}$ and exon 8 (541C1120) reverse primers $^{2}$ as previously described. ${ }^{26}$ The PCR product obtained (approx $1 \mathrm{~Kb}$ ) was digested with the DdeI restriction enzyme - which cleaves the amplicon from SMN2 only - and separated on 2\% agarose gel.

\section{Cell viability}

To determine the sensitivity of fibroblasts and EBV-transformed lymphoblasts to the drugs, cell viability was checked using the XTT method (Roche, Mannheim, Germany). In this assay, metabolically active cells induce the cleavage of the yellow tetrazolium salt XTT to form an orange formazan dye. Cell viability can be determined by quantifying the amount of formazan. The trypan blue exclusion assay was also performed. ${ }^{27}$ Viable (trypan blue-excluding) and dead (trypan blue-stained) cells were counted using a Newbauer chamber (Marienfeld, Germany). The experiments were performed using four different cell lines in each experiment and averaged.

\section{Western blot}

Fibroblasts and EBV-transformed lymphoblastoid cells from SMA patients and control individuals were harvested in RIPA buffer and protease inhibitors to prepare protein extracts (Sigma-Aldrich, St Louis, MO, USA). Subsequently, all samples were quantified by the BCA method (Pierce, Rockford, IL, USA). Western blotting analyses were performed following standard protocols and signal intensity was measured using Chemilmager 5500 (Alpha Innotech, Santa Clara, CA, USA). Further explanation is given in Figure 3 legend. Experiments were performed in triplicate.

\section{Antibodies}

Antibodies used in the present study were: monoclonal antibody anti-SMN (BD Transduction Laboratories, Lexington, KY, USA; 1/5000), anti-GAPDH (Millipore, Temecula, CA, USA; $1 / 10000$ ), anti- $\beta$-tubulin (Sigma-Aldrich; $1 / 300$ ) anti- $\beta$-actin (Sigma-Aldrich; 1/5000) and horseradish peroxidaseconjugated rabbit anti-mouse IgG (DakoCytomation, Copenhagen, Denmark; $1 / 1000)$.

\section{Statistical analysis}

The unpaired $t$-test was performed to analyze differences between control and SMA baseline FL-SMN transcripts, FL/ $\Delta 7$ ratios and protein levels. The paired $t$-test was used to investigate intra-patient differences between fibroblasts and lymphoblasts.

\section{RESULTS}

\section{Origin of the FL-SMN transcripts}

Figure 1 shows that virtually all FL-SMN transcripts of patients harboring the c. 399_402del AGAG mutation originated from SMN2. Similar results were observed in lymphoblasts (data not shown).

\section{Relative quantitative analysis of $S M N$ mRNA and protein in control} and SMA cell lines

As illustrated in Figures $2 \mathrm{a}$ and $\mathrm{b}$, some SMA patients showed diminished FL-SMN mRNA levels in comparison with controls. Others showed similar or even slightly higher values of FL-SMN. 
Sample 1 (type I patient) showed the lowest values in comparison with the remaining SMA samples.

The FL/ $\Delta 7$ ratio was significantly higher in control fibroblasts and lymphoblasts than in patients $(P<0.001)$ (Figures $2 \mathrm{c}$ and $\mathrm{d})$. The
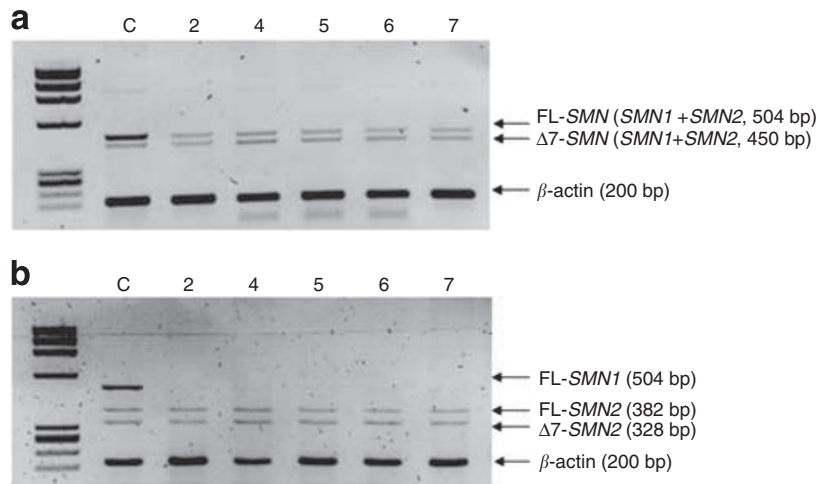

Figure 1 Origin of the FL-SMN transcripts in patients with the c.399_402del AGAG mutation. (a) RT-PCR analysis of total RNA extracted from fibroblasts. The 504-bp fragment includes exons 6-8 of the SMN gene. The 450-bp fragment includes exons 6-8 lacking exon 7 and the 200-bp fragment corresponds to the $\beta$-actin fragment. (b) Ddel digestion of the SMN RT-PCR amplified fragment to determine the origin of the various transcripts. Note that no $S M N$ transcripts originate from the $S M N 1$ gene in these patients. All patients are identified using the same numbers as in Table 1.
$\mathrm{FL} / \Delta 7$ ratio in SMA fibroblasts ranged from 1.13 to 1.73 , whereas in SMA lymphoblasts, it ranged from 1.79 to 2.6. These differences reached statistical significance $(P=0.028)$.

Regarding SMN protein expression, we observed that sample 1 (type I patient) showed the lowest value in comparison with the remaining SMA samples (Figures 2e and $\mathrm{f}$ ).

\section{Relative quantitative analysis of SMN mRNA and protein in response to drugs}

Fibroblasts. When we analyzed the effect of HU and VPA on the FL-SMN transcripts, we observed the maximum increases at $48 \mathrm{~h}$. For PBA, the maximum increases were at $24 \mathrm{~h}$. However, not all cell lines showed changes (Figure 3a). For protein analyses, the highest levels for each cell were mainly reached at $24 \mathrm{~h}$ (Figure $3 \mathrm{c}$ ). Time-fold increases were higher in mRNA than in protein. Table 2 summarizes results that allow each patient to be classified as a responder, a partial responder or a non-responder to each drug, based on their changes in FL-SMN mRNA and protein (positive or absent). All samples considered responders also showed an increase in their FL/ $\Delta 7$ ratios (data not shown) but we did not consider these data as criteria to define the response of a cell line. ${ }^{28}$ If a discrepancy between mRNA and protein was noted, we considered the patient a partial responder. We found two responders (patients 1 and 7) for HU, four (patients 1, 2, 4 and 6) for VPA and two (patients 1 and 4) for PBA. We detected patients responding to all drugs (patient 1), to two drugs (patient 4 ), to one drug (patients 2, 6 and 7) and to none of the three compounds under study (patient 3) (summarized in Table 2). We classified this last
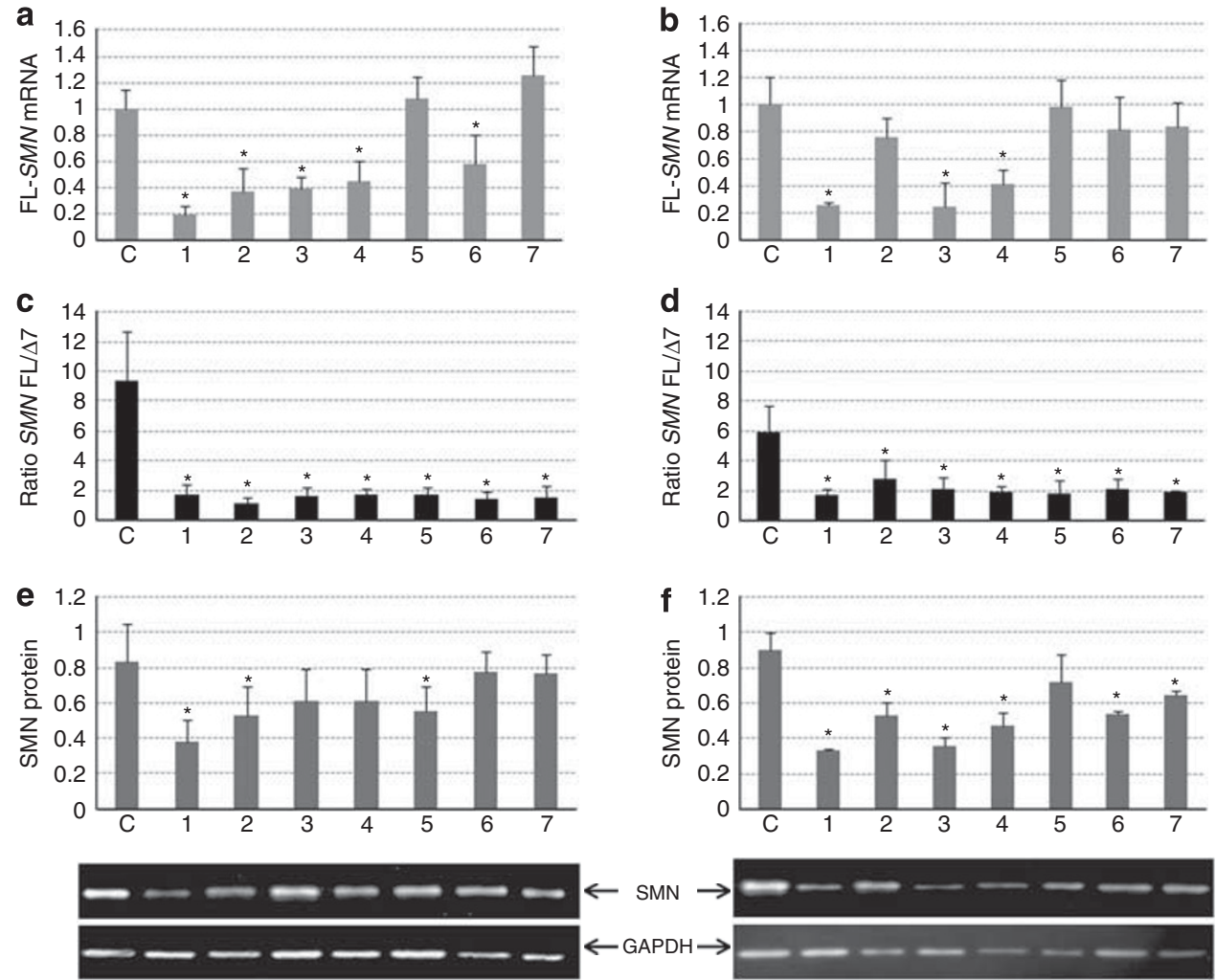

Figure 2 Baseline levels of SMN mRNA and protein. (a) Histogram showing the FL-SMN mRNA in fibroblasts and (b) lymphoblasts relative to control levels determined by the $\Delta \Delta C \mathrm{Ct}$ method. (c) Histogram of FL/ 7 mRNA ratio in fibroblasts and (d) lymphoblasts. (e) Histogram of SMN protein levels in fibroblasts and (f) in lymphoblasts relative to GAPDH as depicted by the respective immunoblots (results with $\beta$-actin and $\beta$-tubulin were similar, data not shown). $\mathrm{C}=$ mean of $\mathrm{C} 1, \mathrm{C} 2$ and $\mathrm{C} 3 ; 1-7=\mathrm{SMA}$ patients. All patients are identified using the same numbers as in Table 1 . 

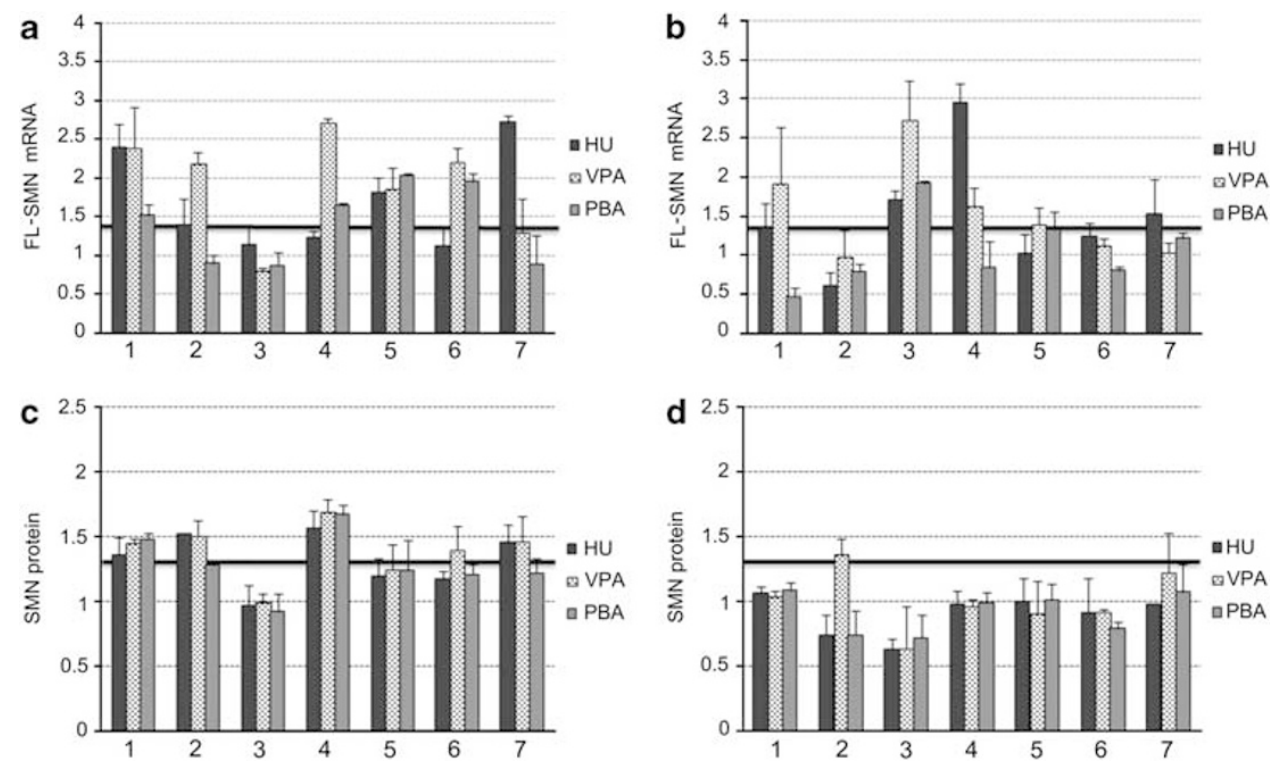

Figure 3 Histograms showing the effect of treatment of seven SMA cells with hydroxyurea (HU), valproic acid (VPA) and phenylbutyrate (PBA). (a) Levels of FL-SMN transcript in fibroblasts and (b) lymphoblasts determined by the $\triangle \Delta C$ t method. We observed variations in GAPDH Ct values ( $\leq 0.5)$ after different biological replicates, resulting in up to 1.4-fold differences. Thus, an increase of mRNA FL-SMN was considered positive when the value obtained was more than 1.4-fold its baseline level. (c) Histogram showing the effect of the same compounds on levels of SMN protein detected by immunoblot in fibroblasts and (d) lymphoblasts. Levels of mRNA and protein are depicted according to the maximum increase observed and are expressed as $n$-fold their baseline set to 1 (continuous line). The highest values of protein were reached at $24 \mathrm{~h}$, with the exception of patient 2, whose peak values for HU and VPA treatment were reached at $48 \mathrm{~h}$, and patient 1 , whose peak values for PBA were reached at $8 \mathrm{~h}$. Owing to the standard deviation observed in basal protein levels $(\leq 30 \%)$ (Figures $2 \mathrm{e}$ and $\mathrm{f}$ ), values higher than 1.3 -fold were considered positive. The thick line represents the threshold for positive response. All patients are identified using the same numbers as in Table 1.

Table 2 Positive (+) or no response (-) of FL-SMN mRNA and SMN protein in fibroblasts after treatment with HU, VPA and PBA

\begin{tabular}{lcccccc}
\hline & \multicolumn{3}{c}{$H U$} & \multicolumn{3}{c}{ VPA } \\
Samples & FL-SMN & Prot & FL-SMN & Prot & FL-SMN & Prot \\
\hline 1 & + & + & + & + & + & + \\
2 & - & + & + & + & - & - \\
3 & - & - & - & - & - & - \\
4 & - & + & + & + & + & + \\
5 & + & - & + & - & + & - \\
6 & - & - & + & + & + & - \\
7 & + & + & - & + & - & - \\
\hline
\end{tabular}

Positive categorical responders (positive concordance between mRNA and protein) are in bold and partial responders (discordance between mRNA and protein) are in normal font. Note the differences between the four sisters (samples 4-7).

patient as a 'complete non-responder'. Interestingly, with a dose increase of one order of magnitude (from 1 to $10 \mathrm{~mm}$ ), fibroblasts from this patient showed a response to VPA (Figure 4), even though they remained unresponsive to $\mathrm{HU}(10 \mathrm{~mm})$ and to the repetitive administration of PBA (every $4 \mathrm{~h}$ ).

We did not observe a correlation between the response to the compound and the number of SMN2 copies. For example, patient 1, who had two SMN2 copies, reached increases that were similar to those for patients $4-6$, who had 4 SMN2 copies. Focusing on the haploidentical sisters (samples 4-7, Table 1) we also observed heterogeneity in their pattern of response. Although patient 7 (non-affected sister) was only a responder for HU, two of her more affected sisters (patients 4 and 6) were responders to VPA. Patient 4 was also a PBA responder, whereas patient 5 (the remaining affected sister) was classified as a partial responder for all compounds (Table 2).
Lymphoblasts. The effect of the different drugs on lymphoblasts is shown in Figures $3 \mathrm{~b}$ and d. In samples 1, 3, 4 and 7, basal FL-SMN levels increased after some treatments, whereas basal protein levels remained unchanged; these patients were, therefore, classified as partial responders in lymphoblasts (Table 3). The remaining samples showed no response in mRNA and protein, regardless of the compound investigated. All samples under treatment with $\mathrm{HU}$ showed a certain increase in $\mathrm{FL} / \Delta 7$ ratios (data not shown). Interestingly, sample 3 - a complete non-responder in fibroblasts - showed lymphoblasts with increments of up to 2.5-fold in FL-SMN after VPA treatment, and increments of up to two-fold in FL-SMN after HU and PBA treatment (Figures $3 \mathrm{~b}$ and $\mathrm{d}$, and Table 3 ).

Cell viability. When SMA cells were cultured in a drug-free medium, the number of cells growing as a monolayer (fibroblasts) or in suspension (lymphoblasts) increased at 24 and $48 \mathrm{~h}$, as observed with trypan blue stain (data not shown). Cell viability was inhibited under experimental doses of the different compounds. As depicted in Figure 5, which illustrates the cell's metabolic state, at doses of $1 \mathrm{~mm}$ for HU and VPA and $2 \mathrm{~mm}$ for PBA, cell viability was decreased by $15-20 \%$ in fibroblasts and by $20-30 \%$ in lymphoblasts after $48 \mathrm{~h}$ of treatment. A dose increment of $\mathrm{HU}$ by one order of magnitude resulted in a $20 \%$ decrease in cell viability in fibroblasts and a $40 \%$ decrease in lymphoblasts. The effect of VPA on cell viability was stronger, decreasing by $50 \%$ in fibroblasts and $75 \%$ in lymphoblasts. We observed no differences between control and SMA cells and no association with the response to the drug treatment (data not shown).

\section{DISCUSSION}

We performed a systematic analysis to determine the level of responses in fibroblasts and lymphoblasts to compounds (HU, VPA, and PBA) 

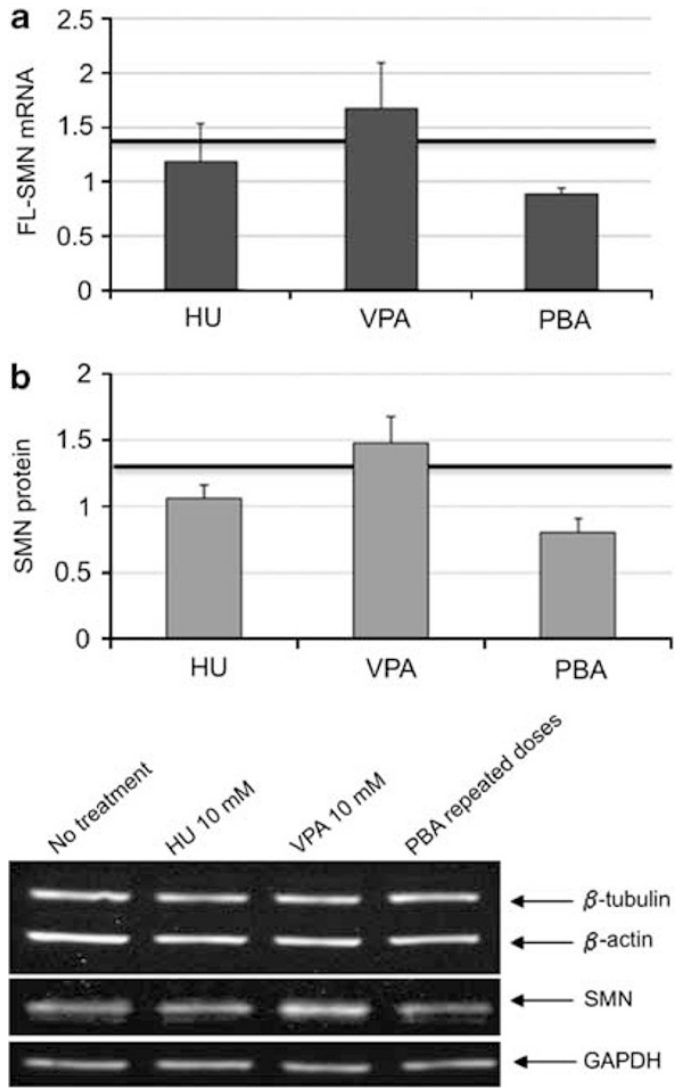

Figure 4 Histograms showing the effect of the increase in dose $(10 \mathrm{~mm})$ of $\mathrm{HU}$ and VPA and the repetitive administration of PBA (2 mM) every $4 \mathrm{~h}$ in fibroblasts of the non-responder patient 3. (a) FL-SMN transcripts expressed as $n$-fold their baseline (set to 1 ). (b) Protein levels relative to $\beta$-actin and $\beta$-tubulin and GAPDH. Note that the increase of SMN expression was achieved only with VPA. The thick line represents the threshold for positive response.

Table 3 Positive (+) or no response (-) of FL-SMN mRNA and SMN protein in lymphoblasts after treatment with HU, VPA and PBA

\begin{tabular}{lcccccc}
\hline & \multicolumn{3}{c}{$H U$} & \multicolumn{3}{c}{ VPA } \\
Samples & FL-SMN & Prot & FL-SMN & Prot & FL-SMN & Prot \\
\hline 1 & - & - & + & - & - & - \\
2 & - & - & - & + & - & - \\
3 & + & - & + & - & + & - \\
4 & + & - & + & - & - & - \\
5 & - & - & - & - & - & - \\
6 & - & - & - & - & - & - \\
7 & + & - & - & - & - & - \\
\hline
\end{tabular}

No categorical responders (positive in mRNA and protein) were observed, only non-responders (negative in mRNA and protein) or partial responders (discordance between mRNA and protein).

that upregulate SMN2 in a group of SMA patients with different genotypes and phenotypes, in particular, in four haploidentical SMA sisters. We found intra- and inter-patient variability even among the sisters, suggesting that tissue and individual factors may affect the response to these compounds.

Levels of FL-SMN in the type I sample were lower than levels from the type II and III samples, in agreement with previous studies. ${ }^{29-32}$
Control samples (with two SMN1 and two SMN2 copies) showed an $\mathrm{FL} / \Delta 7$ ratio of around 6-9, whereas SMA samples showed values ranging from 1.13 to 2.6. Interestingly, in the SMA patients, we found that lymphoblast ratios were slightly higher than those for fibroblasts. These findings may be due to regulation of SMN2 expression in these cells, although the effect of the EBV immortalization on SMN2 regulation is unknown. The FL/ $\Delta 7$ ratio in the SMA cells under study was similar, even though our patients had diverse genotypes and phenotypes. Previous reports have also shown a lack of correlation of $\mathrm{FL} / \Delta 7$ ratios in blood cells, ${ }^{20,28,32}$ fibroblasts ${ }^{16}$ and lymphoblasts. ${ }^{18}$ In the four haploidentical sisters, we found no differences in the FL/ $\Delta 7$ ratios, either in fibroblasts or in lymphoblasts. In fibroblasts from eight discordant families, Helmken et $a^{33}$ found no differences in $\mathrm{FL} / \Delta 7$ ratios between affected and unaffected siblings, although they did find ratio differences in lymphoblasts in one family. The FL/ $\Delta 7$ ratio, therefore, clearly differentiates SMA patients from controls but it is not useful to classify patients according to their severity.

The baseline expression of SMN protein in western blots was lower in SMA samples than in controls. The lowest level was found in the type I patient, in agreement with previous reports. ${ }^{28-30}$ In our remaining patients, there were no noticeable differences even between the discordant sisters, again in concordance with findings in previous studies. $^{4,29}$

Our most significant finding was the intra-and inter-patient variability found in cells exposed to different compounds.

Intra-patient variability was detected after comparison of FL-SMN and protein levels in some patients. Table 2 summarizes patients with a homogeneous response to the drug (either responder or nonresponder) and patients whose response was discrepant (positive mRNA result and no substantial changes in protein or vice versa). We considered these discrepant patients as partial responders. Sumner et $a l^{32}$ reported similar discrepancies in SMA blood samples. They suggested that SMN protein levels might plateau when there are more than two SMN2 copies, as in most of our patients. Patient 3 was considered a categorical non-responder for all three compounds. However, the dose increase by one order of magnitude (which is beyond the therapeutic range for patients) led to a positive response for VPA but not for HU as illustrated in Figure 4. Given that PBA is very toxic at higher doses, we treated the cells of this patient with repetitive PBA doses, ${ }^{15}$ but mRNA and protein levels remained unchanged.

Intra-patient variability was also observed when a patient responded to one drug but not to another. Two of the compounds, VPA and PBA, belong to the same group of HDAC inhibitors (aliphatic acids) and one might presume that their effect on the $S M N 2$ gene would be similar. However, our cells responded differently. The mechanism by which HDACi activate SMN2 expression remains unknown. It is common knowledge that HDACi not only deacetylate histones but also other proteins that are transcriptional regulators. ${ }^{34}$ Two to ten percent of genes are believed to be regulated by histone acetylation and deacetylation, ${ }^{35}$ but we do not know whether these compounds upregulate SMN2 by increasing histone acetylation at the $S M N$ promoter, or by activating the acetylation state of a critical transcription factor. ${ }^{17}$ Kernochan et al ${ }^{36}$ observed that even though acetylated $\mathrm{H} 3$ and $\mathrm{H} 4$ histones predominated in the transcriptional origin of the SMN gene, VPA treatment led to acetylation in farupstream regions where there are usually fewer acetylated $\mathrm{H} 3$ and $\mathrm{H} 4$ histones. Further research will help to clarify the mechanism of action and individual response to these compounds. The third drug investigated, HU, used in sickle cell disease, induces fetal hemoglobin gene expression by stimulating the production of transcription or splicing 

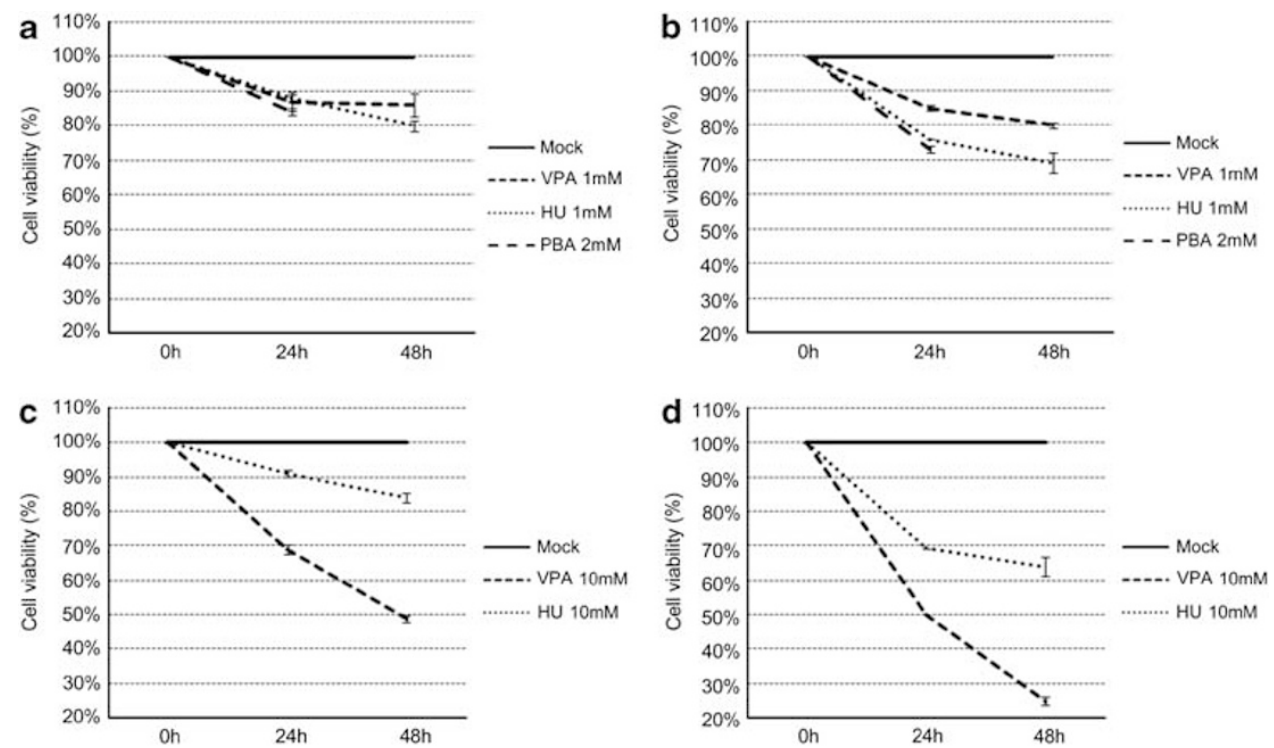

Figure 5 Impact of the different compounds on cell viability determined by XTT assay. (a) Fibroblasts and (b) lymphoblasts treated with HU (1 mM) VPA ( $1 \mathrm{~mm}$ ) and PBA (2 mm); A decrease of around 20-30\% in cell metabolism was observed in both cell lines (lymphoblasts tended to be more sensitive than fibroblasts); (c) fibroblasts and (d) lymphoblasts treated with a dose increase of one order of magnitude. The decrease was around $20-50 \%$ in fibroblasts and $40-80 \%$ in lymphoblasts. Each value is given as mean of four cell lines in three experiments \pm SEM.

factors. Grzeschik et al ${ }^{18}$ proposed that this mechanism could explain the increase in $\mathrm{FL} / \Delta 7$ ratios observed in SMA lymphoblasts. It has recently been suggested that $\mathrm{HU}$ enhances SMN2 expression in lymphoblasts through the release of nitric oxide but its mechanism of action on SMN2 genes is as yet unknown. ${ }^{37}$

In the present study, the three compounds tended to show a different intra-patient response depending on the tissues under investigation. This can be partially explained by the fact that fibroblasts are primary cultures that grow as monolayers, whereas lymphoblasts are EBV-immortalized cells that grow in suspension. Inhibition of cell proliferation has been described in relation to $\mathrm{PBA},{ }^{38,39} \mathrm{VPA}^{40}$ and HU. ${ }^{41}$ In our study, the three compounds inhibited cell growth without noticeable differences between controls and SMA lines. Furthermore, our analysis showed that lymphoblasts were more sensitive than fibroblasts to this effect. Taking the number of viable cells into account may help to normalize RNA or protein values in drug experiments. Lymphoblasts in patient 3 increased their mRNA levels after administration of drugs, whereas their basal protein levels decreased, illustrating the lack of correlation between RNA and protein and the variable range of response in these cells. In this respect, Dayangac-Erden et al ${ }^{42}$ pointed out that EBV-immortalized lymphoblasts are probably not an appropriate cell model to study the upregulation of SMN2.

Inter-patient variability was also notable (Table 2). Four patients were responders to VPA, two to HU and two to PBA. Explanations for this variability in our patients include the molecular characteristics of their SMN2 genes and their individual metabolisms. The number of SMN2 copies has been considered irrelevant for the in vitro positive response. ${ }^{15,17}$ However, their orientation in the SMA locus, their methylation activity, ${ }^{43}$ and the presence of mutations affecting their expression or splicing ${ }^{28,44}$ could influence the final response. Individual metabolism appears to be associated with the differential response to VPA, as previously suggested by Brichta et al. ${ }^{20}$ These investigators studied the level of SMN expression in blood after the oral administration of VPA and found positive, negative and non-responder patients. It is reasonable to hypothesize that $\mathrm{HU}$ and PBA could also have a wide range of response.

SMA patients' cell lines described in previous papers studying SMN2 upregulation had homozygous absence or deletion of the SMN1 gene, whereas most of our patients had a frameshift mutation in SMN1. Our results indicate that pharmacological upregulation of SMN2 may also be a suitable option for patients with point mutations. Indeed, we were able to replicate the results of colleagues who analyzed these compounds separately. However, when we studied a patient concomitantly for the three drugs under analysis, we found a wide range of response. Further testing seems crucial to determine each patient's putative response to these drugs to decide subsequently whether he or she may benefit from a treatment. To date, clinical trials with these compounds have not shown categorical improvements in outcomes and most of these studies conclude the need for homogeneous cohorts of patients. ${ }^{15,20-22,45}$ Selecting patients according to their in vitro response may be a good approach.

Interestingly, our four SMA sisters born to consanguineous parents showed a heterogeneous response to drugs in our experiments (Table 2). We were unable to determine a specific or differential pattern of SMN response based on phenotype. This finding illustrates how variability is present even in patients from the same family, supporting our view that response to each compound should be investigated individually. The variability observed suggests that the positive effects of the drugs are not linked to a direct effect on the SMN2 gene and that their targets are different. This is supported by the fact that the four sisters, with different responsiveness, share the same haplotype that includes the SMN2 gene.

In conclusion, we observed a variable intra-and inter-patient in vitro response in SMA cells exposed to compounds that upregulate SMN2 expression, illustrating the complexity of drug effects. As drug response is dictated by factors beyond $S M N$ genotype, the effect cannot be predicted based on SMN1 mutation or SMN2 copy number alone. To optimize patient stratification in future clinical trials, we suggest primary fibroblasts could be tested before enrolment to 
determine those patients who may respond to the compound under investigation. Garbes et al ${ }^{46}$ have shown that a patient's responsiveness to VPA in whole blood correlated with results in treated fibroblasts. However, in view of the variability that we observed in the cell lines studied a short oral delivery in vivo could be an alternative to better classify the patients. The recent availability of an absolute quantification method for $S M N$ transcripts may help to further categorize patients according to their responder status. ${ }^{47}$ Moreover, in another report, induced pluripotent stem cells generated from skin fibroblasts from a child with SMA were differentiated into motor neurons. ${ }^{48}$ This new model of motor neurons is a promising resource to study SMA disease mechanisms and to further predict the response to drugs that upregulate SMN2 expression.

\section{CONFLICT OF INTEREST}

The authors declare no conflict of interest.

\section{ACKNOWLEDGEMENTS}

This work was supported by FIS05-2416 (EA-R), CIBERER (LA) and GENAME Project (RM-H and SB) and by grants FIS 08-0729 and GENAME Project to EFT. We wish to thank the consenting parents and patients who made this study possible. We are indebted to Carolyn Newey for editing this manuscript, to Ignasi Gich for help with statistics and to Anna Carreras Nolla, Luis Carlos Navas, Lluis Puig and Ana López Ferrer for assistance in obtaining cell lines.

1 Alias L, Bernal S, Fuentes-Prior P et al: Mutation update of spinal muscular atrophy in Spain: molecular characterization of 745 unrelated patients and identification of four novel mutations in the SMN1 gene. Hum Genet 2009; 125: 29-39.

2 Lefebvre S, Burglen L, Reboullet $\mathrm{S}$ et al: Identification and characterization of a spinal muscular atrophy-determining gene. Cell 1995; 80: 155-165.

3 Bussaglia E, Clermont O, Tizzano E et al: A frame-shift deletion in the survival motor neuron gene in Spanish spinal muscular atrophy patients. Nat Genet 1995; 11: 335-337.

4 Cusco I, Lopez E, Soler-Botija C, Jesus Barcelo M, Baiget M, Tizzano EF: A genetic and phenotypic analysis in Spanish spinal muscular atrophy patients with c.399_402del AGAG, the most frequently found subtle mutation in the SMN1 gene. Hum Mutat 2003; 22: 136-143.

5 Martin Y, Valero A, del Castillo E, Pascual SI, Hernandez-Chico C: Genetic study of SMA patients without homozygous SMN1 deletions: identification of compound heterozygotes and characterisation of novel intragenic SMN1 mutations. Hum Genet 2002, 110: 257-263.

6 Lorson CL, Hahnen E, Androphy EJ, Wirth B: A single nucleotide in the SMN gene regulates splicing and is responsible for spinal muscular atrophy. Proc Natl Acad Sci USA 1999; 96: 6307-6311.

7 Monani UR, Lorson CL, Parsons DW et al: A single nucleotide difference that alters splicing patterns distinguishes the SMA gene SMN1 from the copy gene SMN2. Hum Mol Genet 1999; 8: 1177-1183.

8 Gennarelli M, Lucarelli M, Capon F et al: Survival motor neuron gene transcript analysis in muscles from spinal muscular atrophy patients. Biochem Biophys Res Commun 1995; 213: 342-348.

9 Dodds E, Dunckley MG, Roberts RG, Muntoni F, Shaw CE: Overexpressed human survival motor neurone isoforms, SMNDeltaexon7 and SMN+exon7, both form intranuclear gems but differ in cytoplasmic distribution. FEBS Lett 2001; 495: 31-38.

10 Lorson CL, Androphy EJ: An exonic enhancer is required for inclusion of an essential exon in the SMA-determining gene SMN. Hum Mol Genet 2000; 9: 259-265.

11 Cusco I, Barcelo MJ, Rojas-Garcia R et al: SMN2 copy number predicts acute or chronic spinal muscular atrophy but does not account for intrafamilial variability in siblings. J Neurol 2006; 253: 21-25.

12 Feldkotter M, Schwarzer V, Wirth R, Wienker TF, Wirth B: Quantitative analyses of SMN1 and SMN2 based on real-time light cycler PCR: fast and highly reliable carrier testing and prediction of severity of spinal muscular atrophy. Am J Hum Genet 2002; 70: 358-368.

13 Chang JG, Hsieh-Li HM, Jong YJ, Wang NM, Tsai CH, Li H: Treatment of spinal muscular atrophy by sodium butyrate. Proc Natl Acad Sci USA 2001; 98: 9808-9813.

14 Kelly WK, O'Connor OA, Marks PA: Histone deacetylase inhibitors: from target to clinical trials. Expert Opin Investig Drugs 2002; 11: 1695-1713.

15 Andreassi C, Angelozzi C, Tiziano FD et al: Phenylbutyrate increases SMN expression in vitro: relevance for treatment of spinal muscular atrophy. Eur J Hum Genet 2004; 12: 59-65.
16 Brichta L, Hofmann Y, Hahnen E et al: Valproic acid increases the SMN2 protein level: a well-known drug as a potential therapy for spinal muscular atrophy. Hum Mol Genet 2003; 12: 2481-2489.

17 Sumner CJ, Huynh TN, Markowitz JA et al: Valproic acid increases SMN levels in spinal muscular atrophy patient cells. Ann Neurol 2003; 54: 647-654.

18 Grzeschik SM, Ganta M, Prior TW, Heavlin WD, Wang CH: Hydroxyurea enhances SMN2 gene expression in spinal muscular atrophy cells. Ann Neurol 2005; 58: 194-202.

19 Brahe C, Vitali T, Tiziano FD et al: Phenylbutyrate increases SMN gene expression in spinal muscular atrophy patients. Eur J Hum Genet 2005; 13: 256-259.

20 Brichta L, Holker I, Haug K, Klockgether T, Wirth B: In vivo activation of SMN in spinal muscular atrophy carriers and patients treated with valproate. Ann Neurol 2006; 59: 970-975.

21 Liang WC, Yuo CY, Chang JG et al: The effect of hydroxyurea in spinal muscular atrophy cells and patients. J Neurol Sci 2008; 268: 87-94.

22 Swoboda KJ, Scott CB, Reyna SP et al: Phase II open label study of valproic acid in spinal muscular atrophy. PLoS One 2009; 4: e5268.

23 Chen TH, Chang JG, Yang YH et al: Randomized, double-blind, placebo-controlled trial of hydroxyurea in spinal muscular atrophy. Neurology 2010; 75: 2190-2197.

24 Munsat TL, Davies KE: International SMA consortium meeting. (26-28 June 1992, Bonn, Germany). Neuromuscul Disord 1992; 2: 423-428.

25 Burglen L, Lefebvre S, Clermont 0 et al: Structure and organization of the human survival motor neurone (SMN) gene. Genomics 1996; 32: 479-482.

26 Soler-Botija C, Cusco I, Caselles L, Lopez E, Baiget M, Tizzano EF: Implication of fetal SMN2 expression in type I SMA pathogenesis: protection or pathological gain of function? J Neuropathol Exp Neurol 2005; 64: 215-223.

27 Mishell RI, Shiigi JM, Mishell BB, Grabstein KH, Shiigi SM: Prevention of the immunosuppressive effects of glucocorticosteroids by cell-free factors from adjuvantactivated accessory cells. Immunopharmacology 1980; 2: 233-245.

28 Simard LR, Belanger MC, Morissette S, Wride M, Prior TW, Swoboda KJ: Preclinical validation of a multiplex real-time assay to quantify SMN mRNA in patients with SMA. Neurology 2007; 68: 451-456.

29 Coovert DD, Le TT, McAndrew PE et al: The survival motor neuron protein in spinal muscular atrophy. Hum Mol Genet 1997; 6: 1205-1214.

30 Lefebvre $S$, Burlet $P$, Liu $Q$ et al: Correlation between severity and SMN protein level in spinal muscular atrophy. Nat Genet 1997; 16: 265-269.

31 Patrizi AL, Tiziano F, Zappata S, Donati MA, Neri G, Brahe C: SMN protein analysis in fibroblast, amniocyte and CVS cultures from spinal muscular atrophy patients and its relevance for diagnosis. Eur J Hum Genet 1999; 7: 301-309.

32 Sumner CJ, Kolb SJ, Harmison GG et al: SMN mRNA and protein levels in peripheral blood: biomarkers for SMA clinical trials. Neurology 2006; 66: 1067-1073.

33 Helmken C, Hofmann Y, Schoenen F et al: Evidence for a modifying pathway in SMA discordant families: reduced SMN level decreases the amount of its interacting partners and Htra2-beta1. Hum Genet 2003; 114: 11-21.

34 Grozinger CM, Schreiber SL: Deacetylase enzymes: biological functions and the use of small-molecule inhibitors. Chem Biol 2002; 9: 3-16.

35 Pazin MJ, Kadonaga JT: What's up and down with histone deacetylation and transcription? Cell 1997; 89: 325-328.

36 Kernochan LE, Russo ML, Woodling NS et al: The role of histone acetylation in SMN gene expression. Hum Mol Genet 2005; 14: 1171-1182.

37 Xu C, Chen X, Grzeschik S, Ganta M, Wang C: Hydroxyurea enhances SMN2 gene expression through nitric oxide release. Neurogenetics 2011; 12: 19-24.

38 Davis T, Kennedy C, Chiew YE, Clarke CL, deFazio A: Histone deacetylase inhibitors decrease proliferation and modulate cell cycle gene expression in normal mammary epithelial cells. Clin Cancer Res 2000; 6: 4334-4342.

39 McGrath-Morrow SA, Stahl JL: G(1) Phase growth arrest and induction of p21(Waf1/ Cip1/Sdi1) in IB3-1 cells treated with 4-sodium phenylbutyrate. J Pharmacol Exp Ther 2000; 294: 941-947.

40 Jergil M, Kultima K, Gustafson AL, Dencker L, Stigson M: Valproic acid-induced deregulation in vitro of genes associated in vivo with neural tube defects. Toxicol Sci 2009; 108: 132-148.

41 Hammond EM, Green SL, Giaccia AJ: Comparison of hypoxia-induced replication arrest with hydroxyurea and aphidicolin-induced arrest. Mutat Res 2003; 532: 205-213.

42 Dayangac-Erden D, Topaloglu H, Erdem-Yurter H: A preliminary report on spinal muscular atrophy lymphoblastoid cell lines: are they an appropriate tool for drug screening? Adv Ther 2008; 25: 274-279.

43 Hauke J, Riessland M, Lunke S et al: Survival motor neuron gene 2 silencing by DNA methylation correlates with spinal muscular atrophy disease severity and can be bypassed by histone deacetylase inhibition. Hum Mol Genet 2009; 18: 304-317.

44 Bernal S, Alias L, Barcelo MJ et al: The c.859G $>$ C variant in the SMN2 gene is associated with types II and III SMA and originates from a common ancestor. J Med Genet 2011; 47: 640-642.

45 Mercuri E, Bertini E, Messina S et al: Randomized, double-blind, placebo-controlled trial of phenylbutyrate in spinal muscular atrophy. Neurology 2007; 68: 51-55.

46 Garbes L, Riessland M, Holker I et al: LBH589 induces up to 10-fold SMN protein levels by several independent mechanisms and is effective even in cells from SMA patients non-responsive to valproate. Hum Mol Genet 2009; 18: 3645-3658.

47 Tiziano FD, Pinto AM, Fiori S et al: SMN transcript levels in leukocytes of SMA patients determined by absolute real-time PCR. Eur J Hum Genet 2010; 18: 52-58.

48 Ebert AD, Yu J, Rose Jr FF et al: Induced pluripotent stem cells from a spinal muscular atrophy patient. Nature 2009; 457: 277-280. 